\title{
Nanovoid nucleation by vacancy aggregation and vacancy-cluster coarsening in high-purity metallic single crystals
}

\author{
C. Reina, ${ }^{1,2}$ J. Marian, ${ }^{2}$ and M. Ortiz ${ }^{1, *}$ \\ ${ }^{1}$ Division of Engineering and Applied Science, California Institute of Technology, Pasadena, California 91125, USA \\ ${ }^{2}$ Science and Technology Principal Directorate, Lawrence Livermore National Laboratory, Livermore, California 94551, USA
}

(Received 8 March 2011; revised manuscript received 22 June 2011; published 16 September 2011)

\begin{abstract}
A numerical model to estimate critical times required for nanovoid nucleation in high-purity aluminum single crystals subjected to shock loading is presented. We regard a nanovoid to be nucleated when it attains a size sufficient for subsequent growth by dislocation-mediated plasticity. Nucleation is assumed to proceed by means of diffusion-mediated vacancy aggregation and subsequent vacancy cluster coarsening. Nucleation times are computed by a combination of lattice kinetic Monte Carlo simulations and simple estimates of nanovoid cavitation pressures and vacancy concentrations. The domain of validity of the model is established by considering rate-limiting physical processes and theoretical strength limits. The computed nucleation times are compared to experiments suggesting that vacancy aggregation and cluster coarsening are feasible mechanisms of nanovoid nucleation in a specific subdomain of the pressure-strain rate-temperature space.
\end{abstract}

DOI: 10.1103/PhysRevB.84.104117

PACS number(s): 61.72.-y

\section{INTRODUCTION}

This work is concerned with the formulation of a model of nanovoid nucleation at extreme conditions of deformation and temperature such as those found in materials subjected to strong shocks. The aim is to formulate a nucleation model that can be integrated into continuum multiscale descriptions of ductile fracture by void growth and coalescence. Such multiscale models often rely on porous plasticity methods to account for the macroscopic effect of the growing voids, ${ }^{1-13}$ and on micromechanical or localization analyses in order to describe crack growth of the formation of spall planes. ${ }^{5,14-18}$ By design, micromechanical continuum models of porous plasticity either postulate an initial void size and density (e.g., when voids nucleate by decoherence of second-phase particles) or rely on a nucleation model in order to compute the initial void size and density. ${ }^{19,20}$ In this model, nucleation corresponds to the onset of plastic cavitation, i.e., to the formation of voids of a size such that subsequent growth can happen by plasticity. In metals at high tensile pressures, the critical void size for plastic cavitation may be in the nanoscale, in which case plastic cavitation occurs by the emission of discrete dislocation loops. ${ }^{21-24}$

Void nucleation in metals under shock loading has been extensively studied by means of molecular dynamics. ${ }^{25-30}$ While these studies are remarkable for the fidelity and insights that they afford, including the role of inclusions, second-phase particles, dislocations, grain boundaries, and other microstructural features, they often fail to supply an analytical description of nucleation rates that can be effectively integrated into a larger multiscale framework. The present work is primarily concerned with that objective. We specifically consider the homogeneous nucleation of nanovoids by vacancy generation and aggregation. While this is an accepted nucleation mechanism in failure under creep at elevated temperatures, ${ }^{31}$ vacancy diffusion has traditionally been considered to be too slow to operate under dynamic loading conditions. Thus, an ancillary objective of the present work is to investigate the feasibility of diffusion-mediated vacancy aggregation as a nanovoid nucleation mechanism at high tensile pressures and temperatures. Indeed, there is extensive experimental evidence $^{72}$ that, under the extreme conditions of interest here, voids may be nucleated by mechanisms other than the conventional ones of second-phase particle decohesion or grain-boundary cavitation.

Numerical simulations have provided some evidence that vacancy-mediated nanovoid nucleation may be operative homogeneously in single crystals under extreme conditions of tensile pressure and temperature. Strachan et al. ${ }^{32}$ have performed molecular-dynamics simulations of small samples of bcc tantalum and fcc nickel subjected to rapid expansion at $300 \mathrm{~K}$, at pressures in the range of 50-100 GPa induced by impact velocities of $2-4 \mathrm{~km} / \mathrm{s}$. The calculations suggest that large quantities of vacancies are nucleated, followed by rapid coarsening kinetics resulting in the formation of larger voids. As will be shown in Sec. II C, several factors contribute to making vacancy-mediated nanovoid nucleation operative under extreme conditions. First, at very high volumetric deformation the equilibrium concentration of vacancies can be very high, which facilitates their subsequent aggregation. Secondly, vacancy mobility is greatly increased at high temperatures and tensile pressures, which accelerates the aggregation kinetics. Finally, the plastic cavitation of the nanovoids by dislocation emission occurs at comparatively lower pressures and smaller nanovoid sizes at high temperatures owing to the thermal softening of the crystal, which in turn anticipates the nucleation event.

We proceed to quantify these effects by means of several physical models and analysis tools. We begin by estimating typical equilibrium vacancy concentrations in aluminum over the temperature and volumetric deformation range of interest. The orbital-free density functional theory (OFDFT) calculations of Gavini ${ }^{33}$ show that, in this range of volumetric strains, the vacancy-formation energies are exceedingly small or even negative, which suggests that vacancies may be generated nearly spontaneously. We simulate the subsequent diffusion and aggregation of the vacancies by means of a lattice kinetic Monte Carlo (LKMC) approach. ${ }^{34}$ The required vacancy jump rates are computed by transition-state theory with parameters 
obtained from OFDFT calculations. ${ }^{33,35,36}$ In the LKMC calculations, we approximate the total energy of the system by means of an Ising Hamiltonian that considers first and secondnearest-neighbor interactions. The vacancy-cluster kinetics are examined in Sec. II C with particular regard for the effect of temperature and volumetric strain. Finally, we resort to simple continuum estimates of cavitation as a function of pressure and temperature, calibrated to quasicontinuum calculations. ${ }^{21}$ Based on these estimates, together with the LKMC results, we proceed to calculate the nanovoid nucleation times as a function of temperature and volumetric strain. The results of the analysis are discussed in detail in Sec. IV. Indirect comparisons are performed with experimental data of spall tests to test the validity of the model and set meaningful limits to its applicability. The results suggest that, at sufficiently high temperatures and tensile pressures, vacancy aggregation occurs sufficiently fast as to supply an operative nanovoid nucleation mechanism in aluminum over times commensurate with typical shock pulse durations in spall tests. We conclude in Sec. V with a time-continuous description of the nucleation mechanism that can be integrated in a multiscale model of ductile failure.

\section{KINETIC MONTE CARLO SIMULATIONS}

Vacancy diffusion can be simulated accurately by means of molecular dynamics (MD) calculations. ${ }^{37}$ However, MD cannot capture the time scales attendant to vacancy agglomeration and reorganization. A widely used methodology to simulate post-MD diffusive processes is kinetic Monte Carlo (KMC) ${ }^{38-40}$ In KMC, only the discrete jumps of the vacancies are considered, but an a priori knowledge of the transition rates is required. This is not always trivial, as demonstrated by examples found in the literature of complex nonintuitive transitions occurring during surface and bulk diffusion. ${ }^{41,42}$ In addition, failure to consider some physically possible transitions can lead to erroneous kinetics. The KMC rates are commonly obtained by recourse to transition-state theory. ${ }^{43}$ Yet another alternative means of describing vacancy diffusion relies on continuum models. ${ }^{19,44-46}$ Within a continuum setting, the vacancy concentration is thought of as a function of space and time, and evolution equations are used to describe its evolution. Continuum models allow the study of very large systems over long periods of time, albeit at some inevitable loss of fidelity relative to discrete models.

LKMC schemes ${ }^{34,47}$ offer a compromise between accuracy and size of the domain and time span that can be explored computationally, and we have adopted them as the basis for this study. The calculations are based on a secondnearest-neighbor Ising Hamiltonian. The transition rates are estimated by means of transition-state theory and all energies and attempt frequencies are obtained from previous OFDFT calculations. ${ }^{33,35,36}$ The resulting vacancy-cluster kinetics is examined in Sec. II C where particular attention to the effect of temperature and volumetric strain is paid.

\section{A. Physical model}

The model system under consideration consists of a facecentered-cubic (fcc) aluminum crystal containing a random distribution of vacancies at a prescribed temperature, volume, and concentration. The system is analyzed in the canonical $n V T$ ensemble, where $n$ is the number of vacancies, $V$ is the periodic cell volume, and $T$ is the equilibrium absolute temperature of the sample. The state of the system is taken to be characterized solely by the spatial distribution of vacancies on a "frozen" lattice. The relaxation of the atoms surrounding the vacancies is, however, partially taken into account through the energetics considered.

The system is assumed to evolve according to the master equation

$$
\frac{d p_{i}}{d t}=\sum_{j \neq i}\left[r_{j i} p_{j}-r_{i j} p_{i}\right]
$$

where $p_{i}$ is the probability of finding the system in state $i$ and $r_{j i}$ is the transition rate from state $j$ to $i$. The objective of the simulations is to track the diffusion of the vacancies through the lattice and the consequent formation of clusters of various sizes.

In the calculations presented in this paper, Eq. (1) is solved by means of LKMC considering only first-nearest-neighbor random jumps. This is consistent with the migration energetics, which give for an underformed crystal a migration energy of $0.42 \mathrm{eV}$ for first-nearest-neighbor jumps compared to $1.3 \mathrm{eV}$ for second-nearest-neighbor jumps. ${ }^{35}$ The details of the LKMC algorithm and the conditions under which it is valid are well known and here we simply refer the reader to Refs. 34 and 47. However, once a significant fraction of vacancies are in clusters, the kinetics slows down significantly due to "flickering," i.e., rapid transitions between two states that result in slow time evolution but do not alter the overall state of the system. When the system becomes numerically stiff, as in these situations, standard KMC may not be sufficient to advance the time scale to extents necessary to capture nucleation and coarsening. In this context, one must resort to accelerated KMC methods, e.g., based on parallelization. ${ }^{48-51}$ Vacancy diffusion is inherently sequential, and therefore difficult to parallelize. This is because discrete event kinetics advance the time scale independently in different spatial domains, and so reconciling the temporal evolution across the entire subspace partition can be challenging. Here we have opted for the scheme developed by Martinez et al. ${ }^{51}$ which furnishes a synchronous solution to the problem of domain communication. This is achieved by the introduction of the so-called null events, which keep all subdomain internal clocks current in the global sense. In addition, for discrete lattices boundary errors can be controlled by using a sublattice decomposition technique. More details can be found in Martinez et al. ${ }^{52}$

The purpose of the parallel KMC calculations is to ascertain whether the simulation cells used in the serial calculations are sufficiently large to be statistically representative. Several parallel tests were performed over computational cells of varying size, where the cluster number density and size distributions were compared to serial simulations. It was confirmed that, for the time scales of interest in this study, the serial calculations do indeed provide converged statistics and can therefore be used for production runs. 


\section{B. Rate catalog}

The requisite event rates $r_{i j}$ in Eq. (1) are assumed to obey harmonic transition-state theory (HTST). ${ }^{43}$ TST assumes that there exists a critical surface between two neighboring potential wells, with the property that if such a surface is crossed, complete transition occurs. It fails to account for those cases in which an atom crosses the surface and returns before complete transition, and therefore tends to overestimate the true rates. Although dynamical corrections exist to recover the exact rates, ${ }^{53}$ those will not be used in this work. The harmonic assumption, on the other hand, is based on a second-order approximation of the potential energy landscape at the bottom of the energy wells (equivalent to harmonic vibration modes) and at the saddle points in between them. Such approximations tend to be very accurate in solid-state diffusive processes up to at least half the melting temperature, and higher errors are incurred as the temperature increases. ${ }^{47}$

Under these assumptions, the transition rates take the form (see Ref. 54, for instance, for a complete derivation)

$$
r_{i j}=\left\{\begin{array}{cl}
\nu e^{-\beta\left(E_{m}+\Delta E_{i j}\right)} & \text { if } \Delta E_{i j}>0 \\
\nu e^{-\beta\left(\Delta E_{m}\right)} & \text { if } \Delta E_{i j}<0,
\end{array}\right.
$$

where $\Delta E_{i j}=E_{j}-E_{i}$ is the difference in energy between states $i$ and $j, E_{m}$ is the corresponding migration energy, $\nu$ is the attempt frequency, and $\beta=1 / k_{B} T$, where $k_{B}$ is Boltzmann's constant and $T$ is the temperature. The energy of a distribution of vacancies is assumed to be well approximated by an Ising Hamiltonian with first- (1NN) and second-nearestneighbor $(2 \mathrm{NN})$ interactions, namely,

$$
E=-J \sum_{\langle m, n\rangle} \sigma_{m} \sigma_{n}, \quad J=\left\{\begin{array}{cl}
E_{1} & \text { if }\langle m, n\rangle 1 \mathrm{NN} \\
E_{2} & \text { if }\langle m, n\rangle 2 \mathrm{NN} \\
0 & \text { otherwise, }
\end{array}\right.
$$

where $\sigma_{m} \in\{0,1\}$ is the occupation state of site $m$ of the lattice. The calculations presented here use the di-vacancy binding energies $E_{1}$ and $E_{2}$, and the migration energy $E_{m}$, computed by Gavini ${ }^{33,35}$ using zero-temperature quasicontinuum orbital-free density functional theory calculations (QC-OFDFT). As shown in Fig. 1, the divacancy binding energies are positive, which promotes vacancy aggregation and subsequent cluster coarsening. The nearest-neighbor binding energy decreases with volumetric strain, regardless of sign, whereas the second-nearest-neighbor binding energy decreases monotonically with increasing volumetric strain. Therefore, nearest-neighbor binding is dominant under positive volumetric strain (expansion), whereas both nearestand second-nearest-neighbor interactions play a roughly equal role under negative volumetric strain (compression). The migration energy decreases monotonically with increasing volumetric strain, which is expected to accelerate the kinetics. Additionally, the preexponential factor $v$ calculated by $\mathrm{Ho}$ et $a l .{ }^{36}$ using OFDFT is used. Figure 2 shows that the jumpattempt frequency decreases monotonically with increasing volumetric strain, which is expected to slightly decelerate the kinetics.

The number of possible transition rates for a given temperature and volumetric deformation are finite and constitute a rate catalog that can be tabulated and looked up during the simulation.

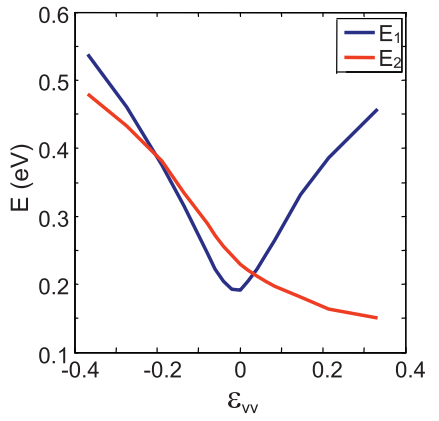

(a)

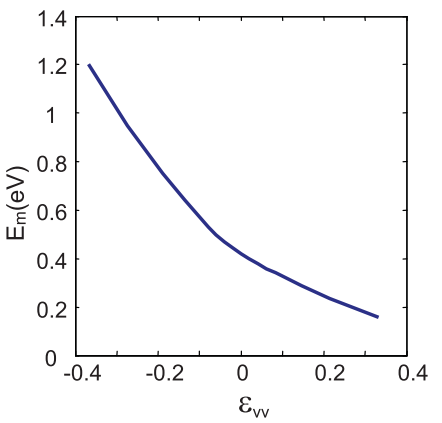

(b)
FIG. 1. (Color online) (a) Divacancy binding energies for first$\left(E_{1}\right)$ and second- $\left(E_{2}\right)$ nearest-neighbor vacancies vs macroscopic volumetric strain. (b) Migration energy vs macroscopic volumetric strain. Figures 6(a) and 9 of Gavini (Ref. 35) on pages 3257 and 3263, respectively. Permission granted from the Royal Society.

\section{Clustering kinetics}

Of primary interest in the present study is the time evolution of vacancy-cluster statistics by size. In particular, the purpose is to ascertain whether nanovoids capable of cavitating plastically can be nucleated in sufficiently short times for the mechanism to operate under shock-loading conditions. A vacancy cluster is defined as a connected component of the graph defined by connecting first- and second-nearestneighbor vacancies. In particular, a cluster of size $l$ is a cluster consisting of exactly $l$ vacancies. It is of note that this working definition of cluster is topological in nature and does not take the geometry of the cluster into account, e.g., whether the cluster is globular or linear.

The time evolution of cluster-size statistics in a $118 \mathrm{~nm}$ cubic periodic cell of fcc aluminum $\left(\sim 10^{8}\right.$ atoms $)$ at $0.1 \%$ concentration ( $\sim 10^{5}$ vacancies $), T=728 \mathrm{~K}$, and $\varepsilon_{v v}=0$ is shown in Figs. 3 and 4. Nominally identical calculations over larger periodic cells using the parallel LKMC algorithm of Martinez et al. ${ }^{51}$ reveal that a periodic-cell size of $118 \mathrm{~nm}$ suffices to provide converged statistics. As expected from the attractive character of divacancy interactions, the cluster-size evolution exhibits an overall trend toward vacancy aggregation

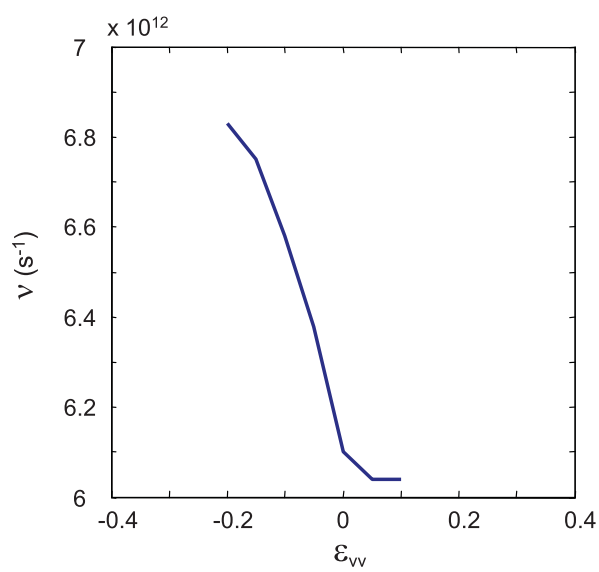

FIG. 2. (Color online) Jump frequency as a function of the macroscopic volumetric deformation. Figure 9 of Ho et al. (Ref. 36) to be supplied by the RSC on signature (Ref. 94). 


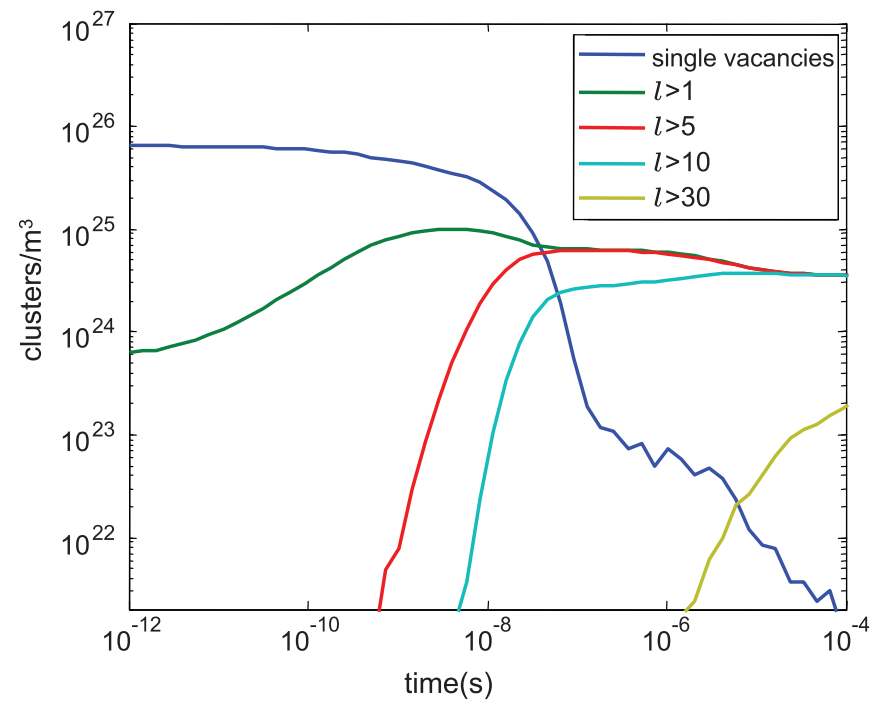

FIG. 3. (Color online) Time evolution of the concentration of clusters larger than size $l$. Computations performed over a $118 \mathrm{~nm}$ cubic periodic cell of fcc aluminum $\left(\sim 10^{8}\right.$ atoms $)$ containing an initial concentration of vacancies of $0.1 \%\left(\sim 10^{5}\right.$ vacancies). $T=$ $728 \mathrm{~K}$ and $\varepsilon_{v v}=0$.

into clusters and a subsequent coarsening of the cluster distribution. Thus, clusters of a certain size appear after an incubation time and their densities initially grow at the expense of smaller clusters, later decreasing as even larger clusters become established. Predictably, the effect of increasing vacancy concentration is to decrease incubation times and accelerate the overall kinetics of aggregation and coarsening, as shown in Fig. 5.

The influence of volumetric strain and temperature on the evolution of cluster statistics up to $1 \mu \mathrm{s}$ is shown in Fig. 6. As expected, temperature accelerates the kinetics, resulting in

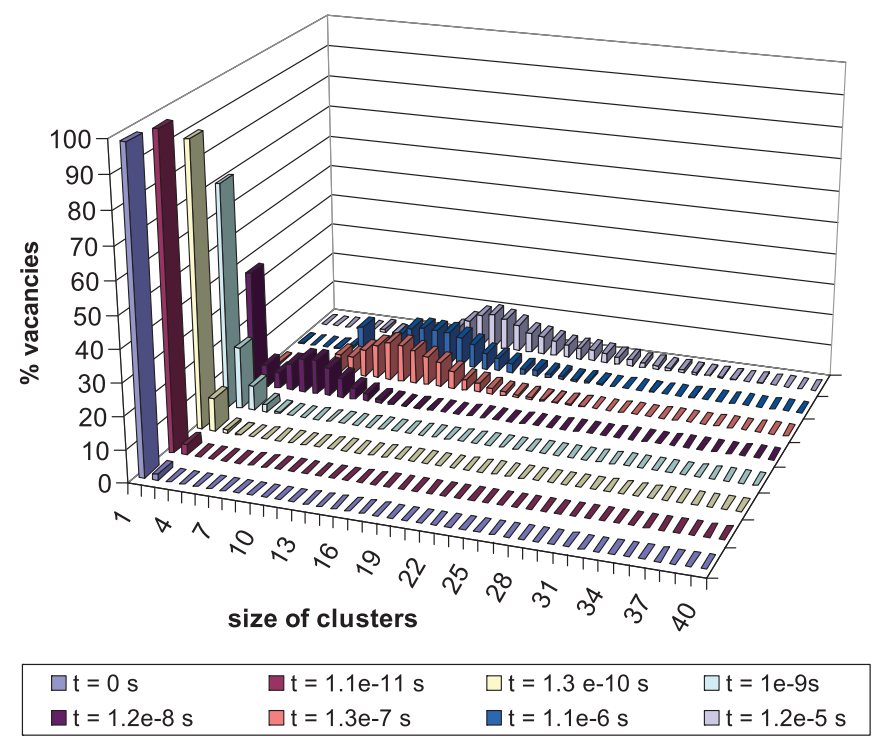

FIG. 4. (Color online) Evolution of histogram of cluster sizes with time. Computations performed over a $118 \mathrm{~nm}$ cubic periodic cell of fcc aluminum $\left(\sim 10^{8}\right.$ atoms $)$ containing an initial concentration of vacancies of $0.1 \%\left(\sim 10^{5}\right.$ vacancies $) . T=728 \mathrm{~K}$ and $\varepsilon_{v v}=0$.

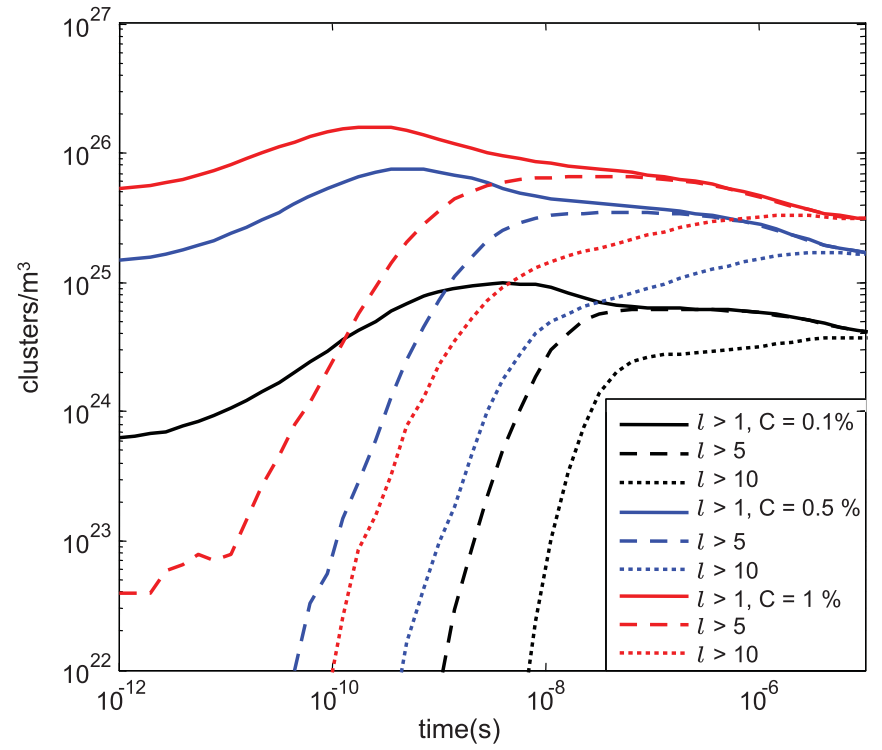

FIG. 5. (Color online) Influence of the concentration on the time evolution of the density of clusters larger than size $l$. Computations performed over cubic periodic cells of fcc aluminum containing $10^{5}$ vacancies. $T=728 \mathrm{~K}$ and $\varepsilon_{v v}=0$.

shorter incubation times and faster cluster coarsening. The net effect of positive volumetric strain (expansion) is also a marked acceleration of the kinetics. Thus, at $900 \mathrm{~K}$, clusters of size 10 nucleate at $\sim 10^{-2} \mu$ s for a volumetric strain of $\varepsilon_{v v}=-0.13$, whereas the same clusters nucleate at around $\sim 10^{-4} \mu \mathrm{s}$ for a volumetric strain of $\varepsilon_{v v}=0.28$, or a two order-of-magnitude acceleration of the kinetics.

\section{NANOVOID NUCLEATION TIMES}

The LKMC calculations summarized in the foregoing reveal that nanovoid nucleation by vacancy aggregation and cluster coarsening is sensitively dependent on both temperature and volumetric deformation. In particular, both temperature and volumetric expansion accelerate the kinetics markedly. In this section, we take the results of preceding LKMC calculations as a basis for estimating nanovoid nucleation times.

We begin by estimating typical vacancy concentrations in aluminum over the range of temperatures and volumetric deformations of interest, $T>800 \mathrm{~K}$ and $\varepsilon_{v v}>0.075$. The calculations of Gavini ${ }^{33}$ show that, in this range, the vacancy-formation energies are exceedingly small or even negative, which suggests that vacancies may be generated nearly spontaneously. This conclusion is in agreement with the molecular dynamics calculations of Strachan et al., ${ }^{32}$ who observed profuse cavitation in shocked metallic samples and showed that such cavitation may be understood as a critical phenomenon. Additionally, experimental observations indicate that, at high pressures, the microstructure evolves toward microtwinning ${ }^{55}$ and slip becomes a secondary mechanism. On the strength of these observations, we neglect the production of vacancy by dislocation activity ${ }^{45}$ and assume that the vacancy concentration is at or near its equilibrium value. 

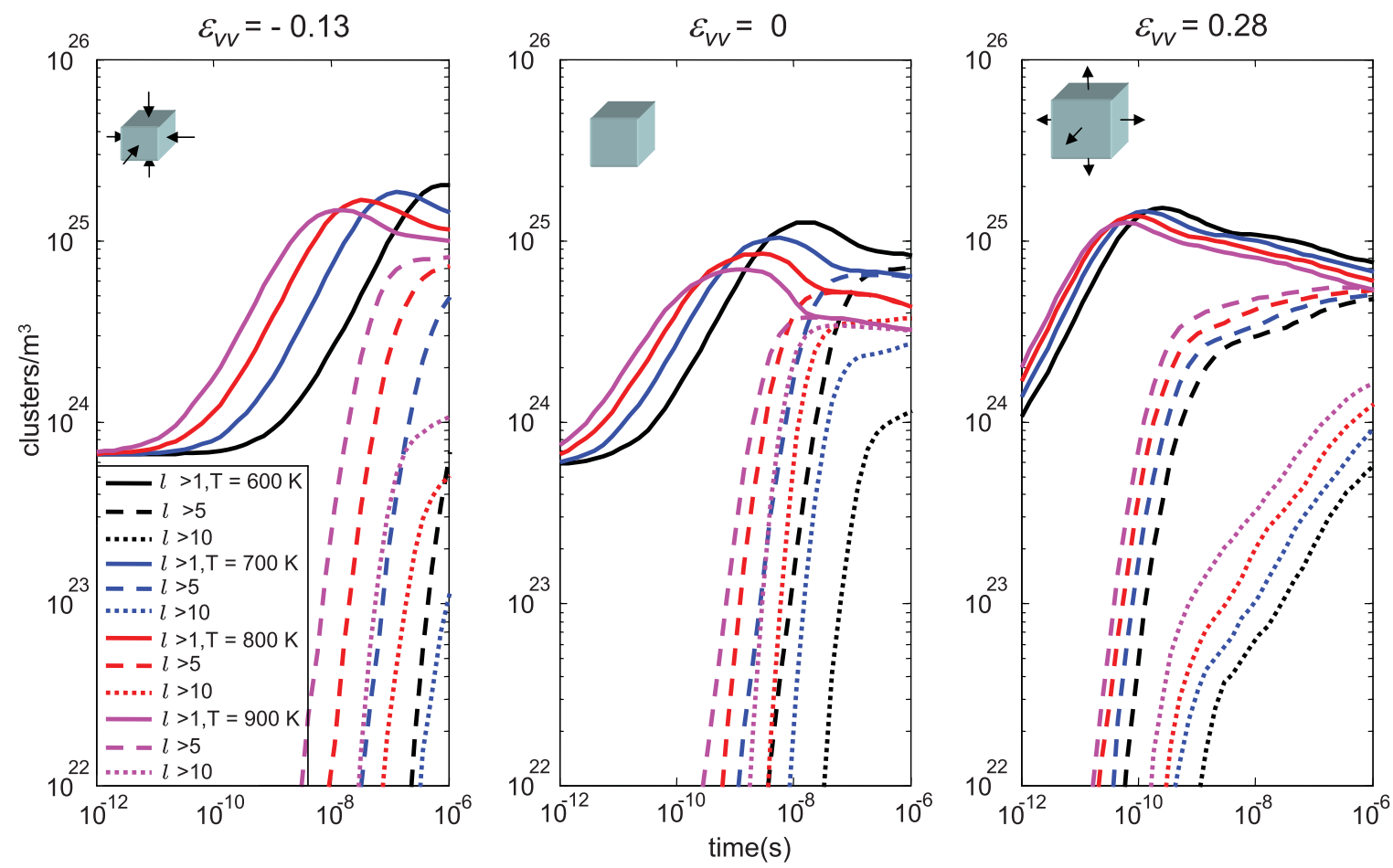

FIG. 6. (Color online) Influence of volumetric strain and temperature on the time evolution of the density of clusters larger than size $l$. Computations performed over a $118 \mathrm{~nm}$ cubic periodic cell of fcc aluminum $\left(\sim 10^{8}\right.$ atoms $)$ containing an initial concentration of vacancies of $0.1 \%\left(\sim 10^{5}\right.$ vacancies $)$.

The equilibrium vacancy concentration follows free-energy minimization. The total free energy per atom as a function of vacancy concentration $c_{v}$ may be estimated as ${ }^{56,57}$

$$
\begin{aligned}
A\left(c_{v}\right)= & c_{v}\left(E_{f v}-T \Delta S_{v}\right) \\
& +k T\left[c_{v} \ln c_{v}+\left(1-c_{v}\right) \ln \left(1-c_{v}\right)\right],
\end{aligned}
$$

where $E_{f v}$ is the vacancy-formation energy and $\Delta S_{v}$ is the change in vibrational energy. This simple form of the free energy presumes a vacancy-formation energy that is independent of vacancy interactions and neglects spatial correlations in the entropic term. The resulting equilibrium concentration of vacancies is

$$
c_{v}=\frac{e^{\Delta S_{v} / k_{B}} e^{-\left(\Delta E_{f v} / k_{B} T\right)}}{1+e^{\Delta S_{v} / k_{B}} e^{-\left(\Delta E_{f v} / k_{B} T\right)}} .
$$

Figure 7 shows the equilibrium vacancy concentration for $E_{f v}$ as computed by Gavini ${ }^{33}$ using OFDFT and for $e^{\Delta S_{v} / k_{B}} \simeq 3$ (see, e.g., Porter and Easterling ${ }^{56}$ ). As may be seen from the figure, the equilibrium vacancy concentration exhibits a sharp upturn at volumetric deformations of the order 0.2 , beyond which the vacancy-formation energy becomes negligibly small.

A nanovoid is said to have been nucleated when it attains the critical size at which it can emit dislocations and subsequently grow by dislocation-mediated plasticity. The process of dislocation emission from nanovoids has been studied by Marian et al. ${ }^{21,58}$ using quasicontinuum calculations. For purposes of the present discussion, a simple continuum estimate of the critical radius for plastic cavitation will suffice. To this end, a void with inner radius $a$ in an infinite medium expanding under an outer tensile pressure $P$ is considered. The material is assumed to obey isotropic von Mises ideal elastoplasticity. Under these assumptions, the critical radius $a_{c}$ for which yielding starts at a given pressure $P$ follows from the relation

$$
P=\frac{2}{3} \sigma_{Y}+\frac{2 \gamma}{a_{c}},
$$

where $\sigma_{Y}$ is the yield stress and $\gamma$ is the surface energy. A derivation of this relation may be found in the Appendix. In

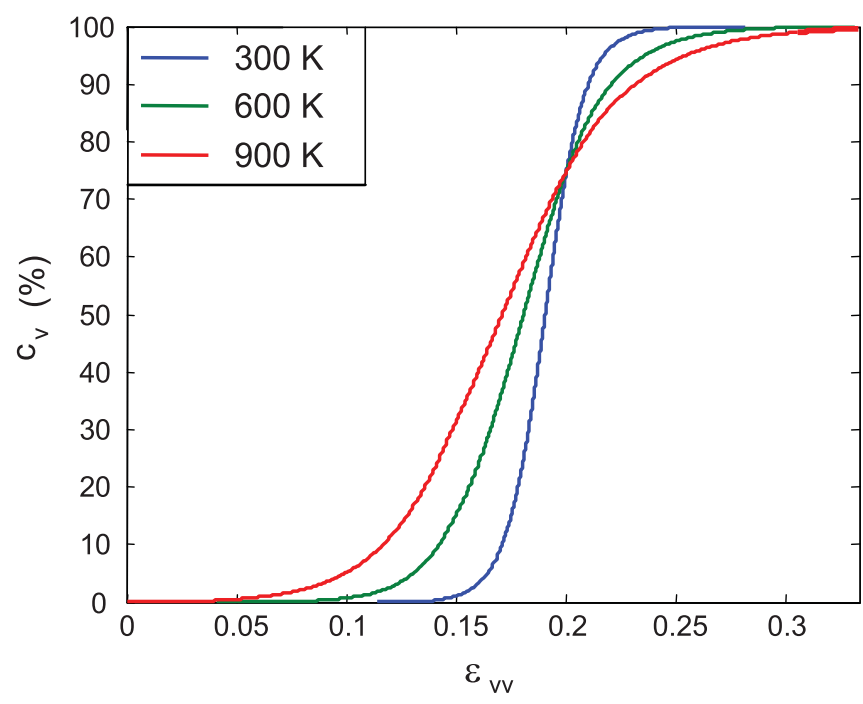

FIG. 7. (Color online) Equilibrium concentration of vacancies vs volumetric strain at different temperatures. 


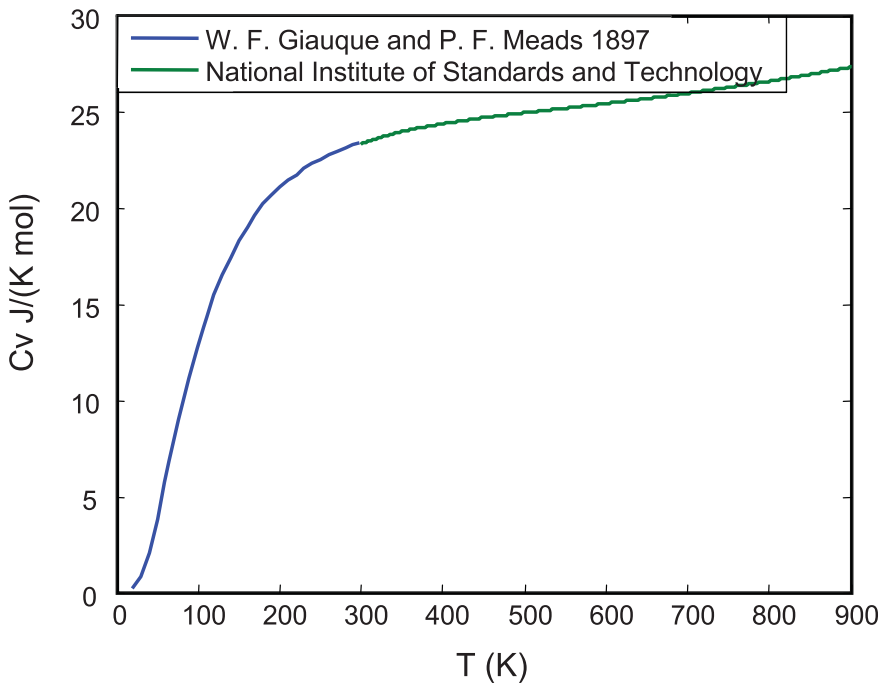

(a)

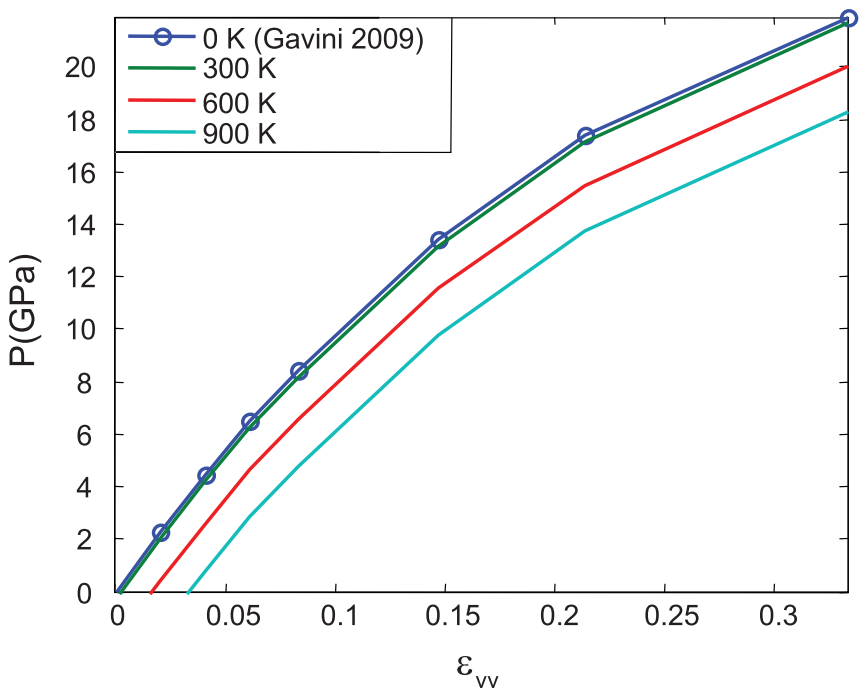

(b)

FIG. 8. (Color online) (a) Heat capacity at constant pressure vs temperature. (b) $0 \mathrm{~K}$ equation of state computed by Gavini ${ }^{63}$ extended to positive temperatures through a Mie-Grüneisen equation of state.

order to account for the temperature dependence of the yield stress, we assume the simple linear thermal-softening relation

$$
\sigma_{Y}=\sigma_{0} \frac{T-T_{m}}{T_{0}-T_{m}}
$$

between the yield stress and the absolute temperature $T$, where $\sigma_{0}$ is the yield stress at the reference temperature $T_{0}$, and $T_{m}$ is the melting temperature. Due to the small sizes of the voids at nucleation time, the attendant dislocation activity is confined to very small volumes. Under these conditions, the strength of the material may be expected to be greatly in excess of bulk macroscopic values. In order to account for this effect, we assume hardness law of the Hall-Petch type

$$
\sigma_{0}=C / \sqrt{a_{c}},
$$

where the constant $C$ is calibrated so as to match the critical volumetric deformation computed by Marian et al. ${ }^{21}$ Similar scaling relations have been used elsewhere to describe nanoscopic plasticity, e.g., at the tip of a nanoindentor. ${ }^{59}$ In the calculations we take $\gamma=0.98 \mathrm{~J} / \mathrm{m}^{2},{ }^{60} T_{0}=0 \mathrm{~K}$, $C=22.77 \mathrm{GPa} \sqrt{\mathrm{nm}}$, and $T_{m}=933.5 \mathrm{~K}$ (Ref. 61) as being representative of aluminum.

In order to relate pressure to volumetric deformation and temperature we use the Mie-Grüneisen equation of state (e.g., Meyers ${ }^{62}$ )

$$
P\left(\epsilon_{v v}, T\right)=P_{0 \mathrm{~K}}\left(\epsilon_{v v}\right)-\frac{\bar{\gamma}}{V} \int_{0}^{T} C_{v}(T) d T .
$$

In particular, we adopt the $0 \mathrm{~K}$ isotherm $P_{0 \mathrm{~K}}\left(\epsilon_{v v}\right)$ computed by Gavini ${ }^{63}$ using OFDFT and set ${ }^{62}$

$$
\left.\frac{\bar{\gamma}}{V} \approx \frac{3 \alpha}{C v \kappa}\right|_{T=298.1 \mathrm{~K}} \approx 2.232 \times 10^{-5} \approx \text { constant } .
$$

We additionally assume that the heat capacity $C_{v}$ at constant volume depends solely on the temperature, Fig. 8(a), and compute it from a $C_{v}-C_{p}$ relation ${ }^{64}$ and experimental values of the heat capacity $C_{p}$ at constant pressure (National Institute of Standards and Technology and Giauque and Meads ${ }^{64}$ ). The resulting equation of state for aluminum is shown in Fig. 8(b).

Figure 9 shows the dependence of the critical cluster size $l_{c}$ on volumetric deformation and temperature predicted by the model just described. As may be seen from the figure, the critical cluster sizes become very small at high temperatures and tensile volumetric strains. A combination of the plastic cavitation model and the LKMC simulations described in the foregoing finally enables the calculation of the times required for the nucleation of a critical nanovoid. The critical times thus predicted for aluminum are shown in Fig. 10. The remarkable conclusion afforded by the figure is that the critical nucleation times can be exceedingly small at high temperatures and tensile volumetric strains. In particular, such critical nucleation times are well within pulse duration times typical of plate-impact experiments. ${ }^{65}$ This in turn is suggestive of the feasibility

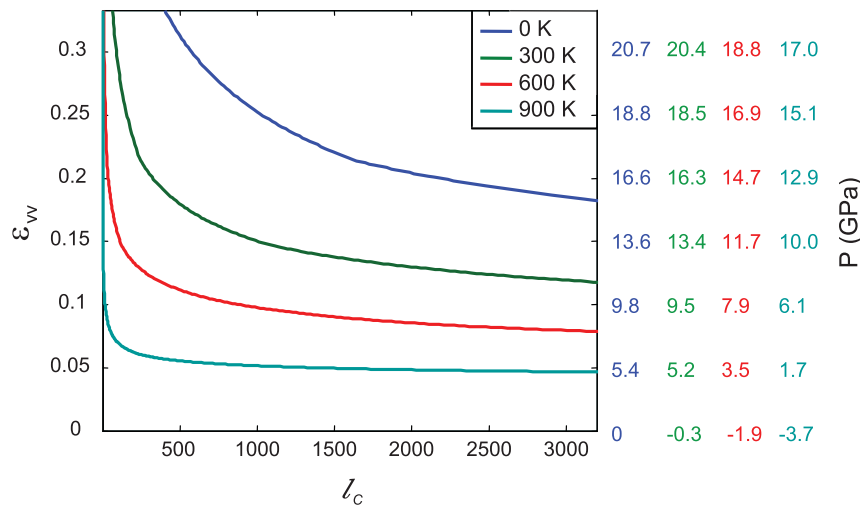

FIG. 9. (Color online) Critical cluster size in aluminum as a function of volumetric strain and temperature. The corresponding pressures at the different temperatures are indicated on the right ordinate axis. 


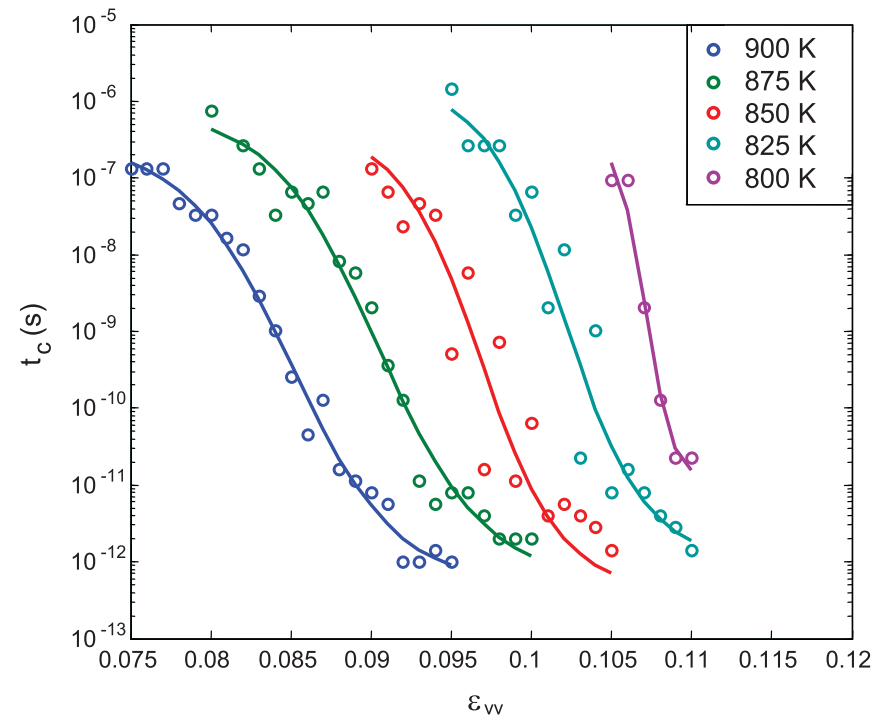

FIG. 10. (Color online) Time required for the formation of critical nanovoids capable of growing by dislocation-mediated plasticity as a function of the volumetric deformation and temperature. Solid lines represent tanh fits of the data.

of diffusion-mediated vacancy aggregation and subsequent vacancy-cluster coarsening kinetics in high-purity metallic single crystals under conditions typical of, e.g., spall tests. Quantitative comparisons with experiments are performed in the following section.

\section{COMPARISON TO EXPERIMENTS}

The experimental validation of the results presented in the foregoing is fraught with difficulty. First, the present study specifically focuses on nanovoid nucleation times by vacancy diffusion in high-purity single crystals under extreme conditions. Therefore, our calculations disregard other types of defects that may also be generated by shocks, thus compounding comparison with experiment. Secondly, the experimental observation of diffusion processes over the time and length scales of interest here is exceedingly challenging. Direct observations of clusters of point defects with atomic resolution have been achieved by Kaiser et al. ${ }^{66}$ using high-angle annular dark-field imaging in a scanning transmission electron microscope (HAADF-STEM). However, these observations pertain to thin films, which compounds the direct comparison with the present analysis. Three-dimensional imaging of voids and their evolution under quasistatic conditions is possible with $\mathrm{x}$-ray tomography. ${ }^{67}$ However, the resolution in this case is restricted to the micrometer range, which is greatly in excess of the length scale of interest in the present study. Despite these difficulties, we proceed to show how the range of validity of the model may be estimated indirectly from the available spall-strength data. To this end, we first compile experimental data for spallation under different conditions of temperature, strain rate, and microstructure. This compilation provides mechanistic understanding indicative of the regime in which the vacancy-aggregation nucleation mechanism can potentially be operative. This analysis is then followed by an estimation of experimental nucleation times from the reported

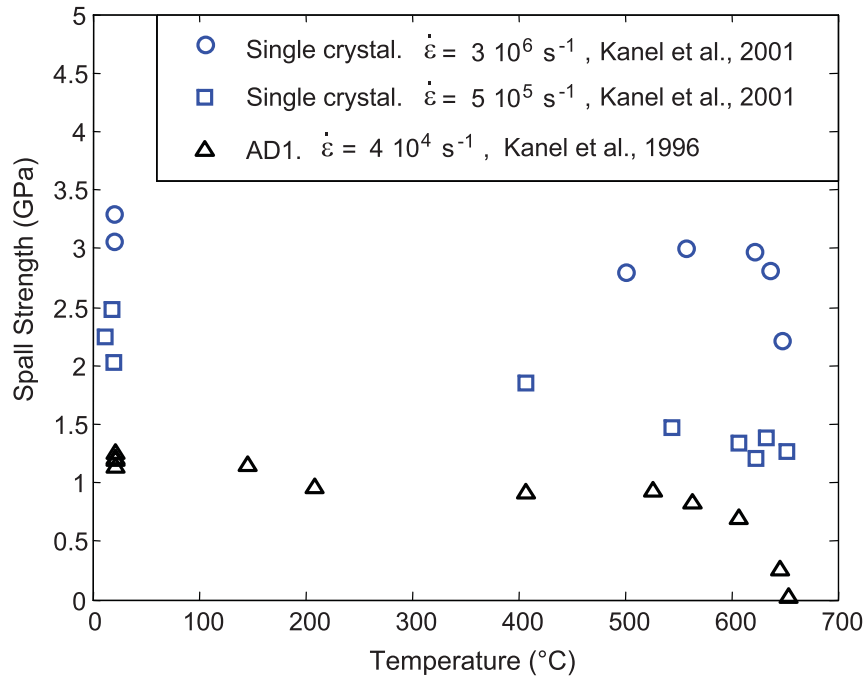

FIG. 11. (Color online) Spall strength as a function of temperature for an aluminum single crystal of $99.999 \%$ purity whose plane is parallel to the (100) crystal plane (Ref. 68) and a polycrystalline aluminum AD1 (wt \% Al: 99.3; Fe: 0.3; Si: 0.3; Cu: 0.05; Mn: 0.025; $\mathrm{Zn:} 0.1$; Ti: $0.1 ; \mathrm{Mg}: 0.05)$ (Ref. 77). The strain rate for each case is indicated in the legend.

spall data. The subsequent quantitative comparison to the predictions of the present nucleation model indeed shows that the times required for the vacancies to aggregate into clusters of critical size are within the same order of magnitude as those inferred from experiments under extreme conditions. Finally, we conclude this section by critically assessing two of the assumptions of the model with a view to further bracketing its limits of validity.

The main property measured in spall experiments is the maximum strength attained during the failure process, which is referred to as spall strength. Results of these measurements from a variety of sources are compiled in Figs. 11 and 12. The influence of temperature, microstructure, and strain rate on spall strength, and the corresponding underlying mechanisms, may be summarized as follows.

Influence of the temperature. Figure 11 shows that, for moderate strain rates $\left(\sim 10^{6} \mathrm{~s}^{-1}\right)$, the dynamic strength is fairly independent of temperature except close to the melting point, at which point the spall pressure decreases dramatically. ${ }^{68}$ Similar conclusions were also obtained from semiempirical equations of state by Moshe et al. ${ }^{69}$ This strong thermal sensitivity in the high temperature range provides a strong indication that the experimentally observed behavior is in the thermally activated range, which is precisely the range considered in the model. However, as will be shown subsequently, the strain rates considered in these experiments are well below the range of applicability of the model and therefore no direct comparison can be performed with these data. This gap notwithstanding, we may note that the pressure variation in the simulations for the temperature range considered ( $800-900 \mathrm{~K}$ ) is $\simeq 40 \%$, which is not unlike the observed experimental values of $\simeq 0 \%, 25 \%$, and $65 \%$ for the three samples considered in the figure.

Influence of the microstructure. At low-to-moderate strain rates $\left(\sim 10^{4}-10^{6} \mathrm{~s}^{-1}\right)$, the spall strength is observed to be strongly dependent on the microstructure ${ }^{70}$ (cf. Figs. 11 


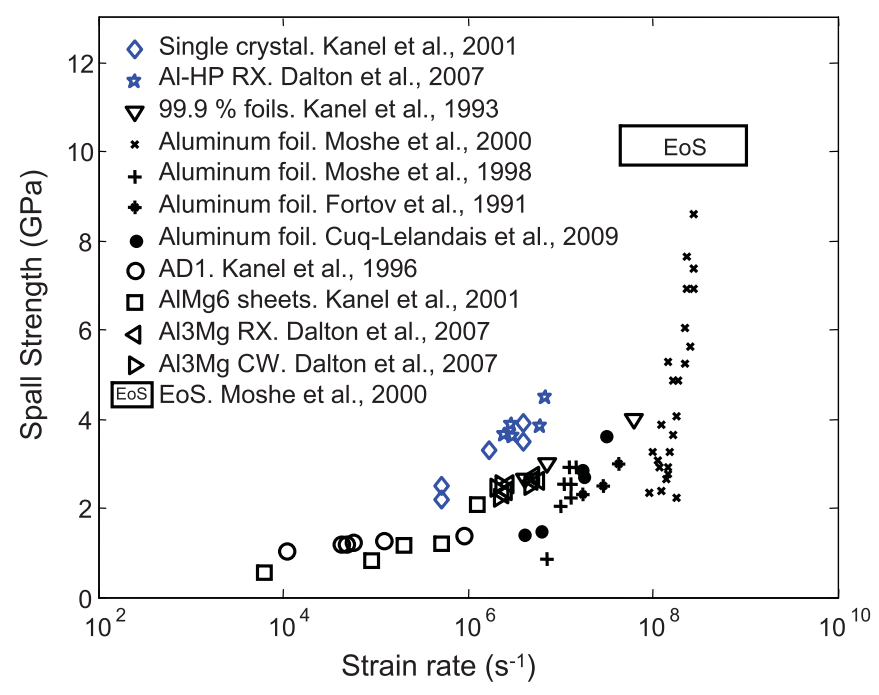

FIG. 12. (Color online) Strain rate dependence of the spall strength for aluminum samples of different purity. ${ }^{68,69,74,75,77-80}$ The theoretical spall strength calculated by Moshe et al. ${ }^{69}$ from the equation of state is also represented in the figure.

and 12). In general, the spall strength increases with increasing grain size, with the maximum spall strength being attained for single crystals. ${ }^{68,71,72}$ This trend is owed to the ability of grain boundaries to act as nucleation sites. In the case of single crystals, the spall strength is also observed to be sensitive to the crystal orientation. ${ }^{73}$ Second-phase particles are weak points of the material that may also act as preferential locations for void nucleation. Experiments indeed indicate that inclusions lower the spall strength, the particle spacing being an important parameter. $^{72}$

Influence of the strain rate. Figure 12 additionally shows a monotonic increase of the spall strength with strain rate. Two explanations can be provided to justify this behavior. A first plausible explanation of this trend rests on the observation that processes of damage accumulation have more time to operate at lower deformation rates, thereby resulting in smaller spall strengths. The second-not unrelated-explanation of the trend is suggested by experimental observations of a change in initiation mechanism with strain rate. ${ }^{71}$ Thus, experiments show that spall failure in polycrystalline materials changes from intergranular and transgranular to purely transgranular as the strain rate increases. ${ }^{72,74,75}$ In the limiting case of extremely high strain rates, initial flaws and defects present in the system do not have time to operate and nucleation is believed to occur almost simultaneously throughout the spall plane, the theoretical limit thus being reached. ${ }^{72}$ Such limit, known as ultimate spall strength, can be estimated as ${ }^{76}$

$$
P_{\text {th }}=\sqrt{\frac{U_{\mathrm{coh} B_{0}}}{8 v_{0}}},
$$

where $v_{0}$ is the specific volume at zero pressure, $U_{\text {coh }}$ is the specific cohesive energy, and $B_{0}$ is the bulk modulus at zero pressure. For aluminum, this expression yields $17.1 \mathrm{GPa}$, which is therefore expected to be an upper bound on the experimentally observed spall strength. ${ }^{76}$ Moshe et al. ${ }^{69}$ have also estimated the theoretical spall strength from equation of states for aluminum obtaining values within the range
$10.1-10.8 \mathrm{GPa}$ for temperatures in the range of 300-600 K. This range is shown in Fig. 12, labeled as "EoS," and is close to the value of $8 \pm 1 \mathrm{GPa}$ experimentally obtained by Moshe et al $^{69}$ using a $20 \mathrm{ps}$ pulse high-power laser.

We note that most of the measurements of spall strength $P_{\text {spall }}$ and strain rates alluded to in the foregoing were obtained from back-surface measurements as

$$
P_{\text {spall }}=\frac{1}{2} \rho c \Delta u
$$

and

$$
\dot{\epsilon}=\frac{\Delta u}{\Delta t} \frac{1}{2 c},
$$

respectively, where $\rho$ is the density, $c$ is the bulk sound speed, $\Delta u$ is the velocity jump, and $\Delta t$ is the time interval associated with the pullback signal (see, for instance, Fig. 2 in Chen et $a l .^{73}$ ). The interval $\Delta t$, referred to as critical time of spallation by Hanim and Klepaczko, ${ }^{81}$ provides an estimate of the time over which the material in the spall plane is in tension before incipient spall occurs. This time accounts for the nucleation of voids, their growth and partial coalescence, although nucleation is thought to be the ratelimiting mechanism controlling failure. ${ }^{68}$ On the strength of these observations, here we take $\Delta t$ to be a measure of the actual time for nucleation. However, further computational and experimental work is required in order to validate this assumption in the temperature and pressure range examined in this study. The nucleation times corresponding to the experimental data shown in Fig. 12 are thus estimated as $\Delta t=P_{\text {spall }} / 2 \rho c^{2} \dot{\epsilon}$ and shown in Fig. 13. For this conversion, we use the values $c=5.4 \mathrm{~km} / \mathrm{s}$ and $\rho=2.7 \mathrm{~g} / \mathrm{cm}^{3}$ from Brewer et al. ${ }^{72}$ Figure 13 also includes the nucleation times predicted by the model (cf. Fig. 10), expressed as a function of pressure.

A salient feature of the experimental data that is in contrast to the model predictions is the presence of an asymptote at a spall time of $10^{-10} \mathrm{~s}$, or at a strain rate of $10^{8} \mathrm{~s}^{-1}$ (cf. Fig. 12). In addition, the experimental values of $\Delta t$ may be expected to constitute a lower bound to the nucleation times predicted by the model, since several other physical mechanisms may potentially contribute to the nucleation of voids in engineering materials. These discrepancies set limits to the applicability of the present model, which we proceed to investigate. We begin by noting that the equilibrium vacancy concentration postulated by the model requires a certain time to become established. Assuming that vacancies are created through the formation of a stable interstitial-vacancy pair, the equilibration time can be estimated as

$$
t_{f}=\frac{c_{v}}{v_{0} e^{-\left(\Delta E_{f v}+\Delta E_{f i}\right) / k_{B} T}},
$$

where $v_{0}=5.16 \times 10^{13} \mathrm{~Hz}$ is the Debye frequency ${ }^{82}$ and $\Delta E_{f i}$ is the energy of formation of an interstitial atom. This relation assumes that the energy of formation of a vacancy-interstitial pair can be approximated as the sum of the individual formation energies, and that the migration energy associated with the movement of interstitials is negligible. In order to ascertain the dependence of $t_{f}$ on pressure and temperature, the corresponding dependence of $\Delta E_{f i}$ and $\Delta E_{f v}$ needs to be known. The latter has been computed by 


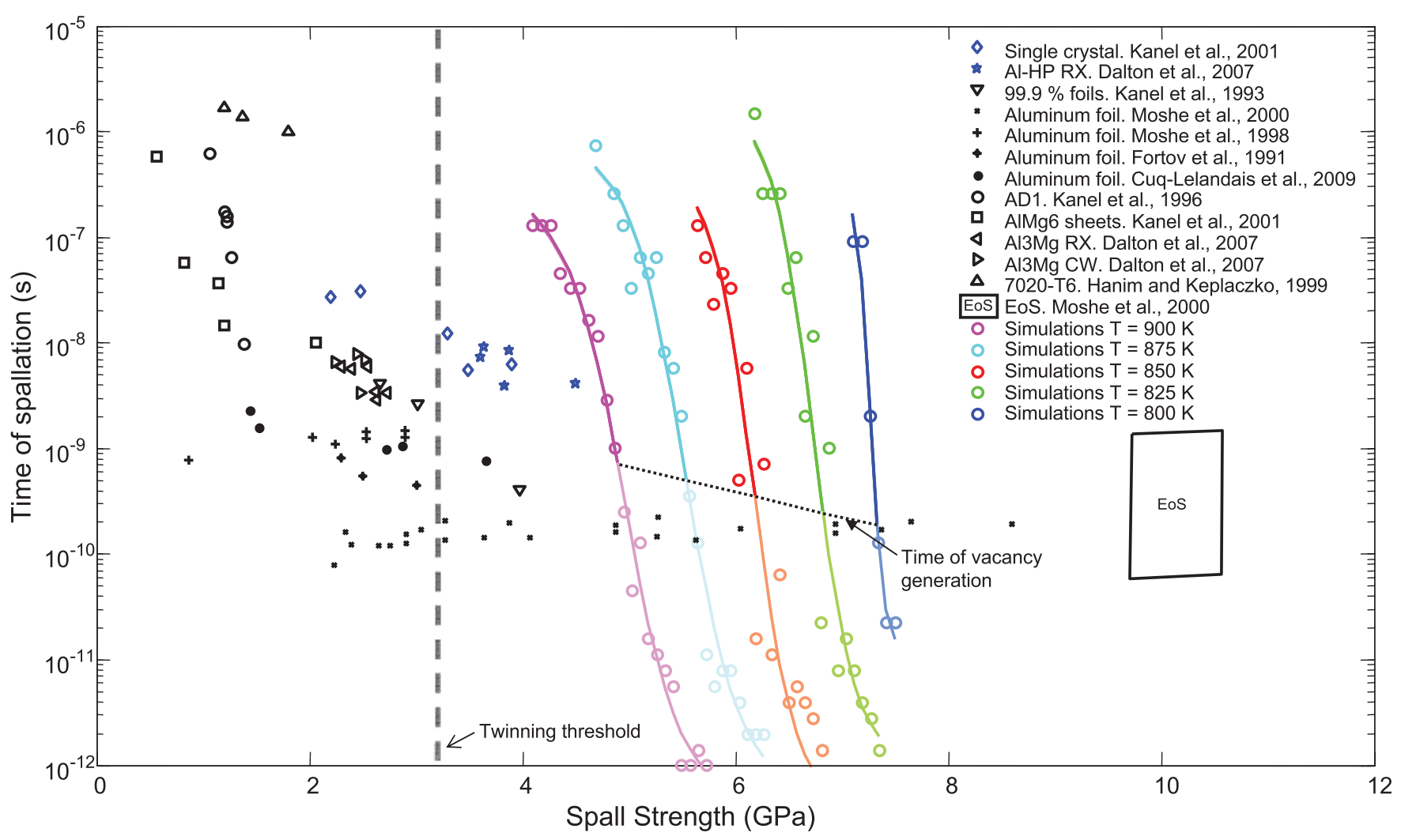

FIG. 13. (Color online) Comparison between the nucleation times predicted by the model as a function of pressure and estimations of the experimental and theoretical times of spallation. Here, we represent the computed nucleation times given in Fig. 10 as a function of pressure instead of volumetric deformation. On the other hand, the experimental spallation times have been obtained by converting the pressure-strain-rate data of spall (Refs. $68,69,74,75,77-81)$ using the formula $\Delta t=P_{\text {spall }} / 2 \rho c^{2} \dot{\epsilon}$. The theoretical spall-strength data obtained by Moshe $e t$ al. ${ }^{69}$ are also represented in the figure using the same conversion. Finally, two additional bounds have been included in the figure that set the limits of applicability of the nucleation model: the twinning stress threshold as a lower pressure bound, and the time of formation of the equilibrium concentration of vacancies as a lower bound on the nucleation times.

Gavini. ${ }^{33}$ However, the pressure and temperature dependence of $\Delta E_{f i}$ does not appear to be available in the literature. Assuming, for want of a better model, that $\Delta E_{f i} \approx 2 \Delta E_{f v},{ }^{83}$. then Eq. (5) gives

$$
t_{f}=\frac{1}{v_{0}} \frac{3 e^{2 \Delta E_{f v} / k_{B} T}}{1+3 e^{-\left(\Delta E_{f v} / k_{B} T\right)}} .
$$

A dashed line joining the values obtained in this fashion for each temperature is shown in Fig. 13. This line sets a lower bound on the applicability of the model and lies, as expected from the preceding arguments, above the experimental times. Incidentally, this bound compares remarkably well with the experimental values of spall strength for single crystals at the lower end of the pressure range under consideration, and with the ultimate spall strength at the upper end. Although the highest experimental spall pressures do not correspond to highpurity single crystals such as considered here, the spall stress for different microstructures may be expected to converge at very high strain rates to a common structure-independent ultimate spall strength, ${ }^{32}$ since voids formed at existent defects and inhomogeneities do not have time to grow and coalesce, and homogeneous nucleation dominates. ${ }^{69}$ This is precisely the regime where vacancy aggregation may be expected to be the rate-limiting nucleation mechanism.

To further define the range of validity of the model, we next establish a lower bound in terms of pressure. To this end, we proceed to ascertain the conditions under which vacancies are in thermodynamic equilibrium, i.e., when the concentration of vacancies in the system can be approximated by Eq. (5). At relatively low strain rates and moderate stresses, plastic slip may act as a significant source of vacancies through the jog dragging mechanism. However, studies have shown that, under strong shocks, the dislocation microstructure experiences a sharp increase in density, resulting in sessile dislocation networks. ${ }^{84,85}$ Beyond this point, in most metals plasticity is known to proceed by twinning, ${ }^{55,86,87}$ which effectively renders dislocation movement inoperative as a vacancy source. Indeed, there is ample experimental evidence of twinning in nanocrystalline aluminum specimens. ${ }^{88}$ For single crystals, the literature is more sparse, with some studies suggesting twinning under extreme conditions. ${ }^{89}$ The transition between slip and twinning occurs when the activation stress for twinning is lower than that needed to unlock the dislocation microstructure. This transition is known to depend on temperature, strain rate, and grain size. ${ }^{87}$ The twinning stress threshold, however, is insensitive to temperature and is 
expected to decrease with the strain rate. ${ }^{85}$ Both analytical models and experiments are suggestive of a shear stress for twinning smaller than $1.5 \mathrm{GPa} .{ }^{85,90}$ An order of magnitude for the corresponding pressures may be obtained simply by assuming a uniaxial-strain state of stress: $\sigma_{11}=3 \frac{1+v}{1-v} P$, $\sigma_{22}=\sigma_{33}=3 \frac{v}{1+v} P$. In addition, assuming impact on a (100) crystal, the shear stress in each of the eight activated slip systems is $\frac{3}{\sqrt{2}} \frac{(1-2 v)}{1+v} P=0.47 P$. This relation gives a pressure threshold for twinning of about $3.2 \mathrm{GPa}$, which is indeed below the pressure range considered in the simulations (cf. Fig. 13).

\section{DISCUSSION}

Figure 13, which shows the predicted time required for the formation of critical nanovoids as a function of pressure and temperature, may be regarded as the main outcome of the present work. In the range of validity of the model in $T-P-\dot{\epsilon}$ parameter space, the critical nucleation times are exceedingly small, allowing for diffusion-mediated vacancy aggregation and subsequent vacancy cluster coarsening to be feasible operating mechanisms of nanovoid nucleation. In addition, the tanh fit shown in Fig. 13 matches the results of the calculations quite closely and can, therefore, be used as an analytical model of nanovoid nucleation for pulses consisting of ostensibly constant temperature and tensile pressure. In particular, the nucleation model determines the onset of porous plasticity as described, e.g., by a continuum model, and supplies the initial void size $a_{0}=a_{c}$ and the estimate $f_{0} \approx c_{v}$ for the initial void fraction. The model can be extended to transient conditions simply by applying it in rate form. To this end, we may define a void expansion speed as

$$
v(p, T)=\frac{a_{c}(p, T)}{t_{c}(p, T)},
$$

where $a_{c}(p, T)$ and $t_{c}(p, T)$ denote the critical void radius and nucleation time at constant pressure and temperature, respectively. For transient conditions we may then write

$$
a(t)=\int_{0}^{t} v(p(\tau), T(\tau)) d \tau .
$$

Plastic cavitation occurs when the void sizes attain a critical value, i.e.,

$$
a(t)=a_{c}(p(t), T(t)) .
$$

This relation can again be used to estimate the onset of porous plasticity, and the simple estimates $a_{0}=a_{c}$ and $f_{0} \approx c_{v}$ again supply the requisite initial conditions for continuum models.

In closing, we point out that kinetic Monte Carlo models such as described in the foregoing have been extensively used to simulate vacancy aggregation in metals. ${ }^{39,91}$ These models suffer from several limitations, most notably, the rigid lattice approximation, which neglects elastic interactions between vacancies; the simplified Ising Hamiltonian, which tends to break down for complex cluster geometries and at void surfaces; and limitations attendant to the use of harmonic transition-state theory. Because of these and other limitations, the results presented in this paper cannot be considered predictive and mainly provide a preliminary assessment as to the feasibility of nanovoid nucleation by vacancy aggregation at high tensile pressures and temperatures. A number of improvements of LKMC simulations have been proposed (e.g., Dai et $a l .{ }^{92}$ ) which provide avenues for further improvement of the model.

\section{ACKNOWLEDGMENTS}

The authors gratefully acknowledge the discussions with V. Gavini, S. Serebrinsky, D. C. Swift, and J. McNaney, as well as Enrique Martinez, author of the parallel LKMC code used in the calculations. Support for this study was provided by the Department of Energy National Nuclear Security Administration under Award No. DE-FC52-08NA28613 through Caltech's ASC/PSAAP Center for the Predictive Modeling and Simulation of High Energy Density Dynamic Response of Materials. This work was performed under the auspices of the U.S. Department of Energy by Lawrence Livermore National Laboratory under Contract No. DE-AC52-07NA27344.

\section{APPENDIX: CRITICAL PRESSURE FOR PLASTIC VOID CAVITATION}

In this Appendix, a preexistent spherical void of radius $a$ in an infinite medium is considered, and the required stress applied at infinity in order for plasticity to initiate at the surface of the cavity is computed. The theory of continuum mechanics is used in order to obtain such an estimate.

Due to the spherical symmetry, the stresses obey the following equilibrium and compatibility equations in spherical coordinates:

$$
\begin{gathered}
\frac{d \sigma_{r r}}{d r}-\frac{2}{r}\left(\sigma_{\theta \theta}-\sigma_{r r}\right)=0, \\
\frac{d}{d r}\left(\sigma_{r r}+2 \sigma_{\theta \theta}\right)=0,
\end{gathered}
$$

which have as general solution

$$
\begin{gathered}
\sigma_{r r}=A+\frac{B}{r^{3}}, \\
\sigma_{\theta \theta}=A-\frac{B}{2 r^{3}} .
\end{gathered}
$$

$A$ and $B$ are constants to be determined by the boundary conditions. The stress imposed at infinity is $\sigma_{r r}(r \rightarrow \infty)=P$, while the effect of the surface energy $\gamma$ on the inner surface is proven in the following to be $\sigma_{r r}(r=a)=\frac{2 \gamma}{a}$. The resulting stresses then are

$$
\begin{aligned}
& \sigma_{r r}=P\left(1-\frac{a^{3}}{r^{3}}\right)+\frac{2 \gamma}{a} \frac{a^{3}}{r^{3}}, \\
& \sigma_{\theta \theta}=P\left(1+\frac{a^{3}}{2 r^{3}}\right)-\frac{\gamma}{a} \frac{a^{3}}{r^{3}} .
\end{aligned}
$$

Applying the von Mises yield criterion, plasticity will occur in the inner surface when $\sigma_{\theta \theta}(a)-\sigma_{r r}(a)=\sigma_{Y}$. Equivalently,

$$
P=\frac{2}{3} \sigma_{Y}+\frac{2 \gamma}{a},
$$

which is the desired relation.

In order to obtain the pressure at the surface of the cavity $\left[\sigma_{r r}(r=a)=\frac{2 \gamma}{a}\right]$, the matrix surrounding the void is first assumed to be finite with radius $b$ and made of isotropic homogeneous material. The desired analytical result is then 
evaluated as the external radius and the stiffness of the material tend to infinity. A Hookean constitutive law with parameters $\lambda$ and $\mu$ is used

$$
\begin{gathered}
\sigma_{r r}=\lambda\left(\varepsilon_{r r}+\varepsilon_{\theta \theta}+\varepsilon_{\phi \phi}\right)+2 \mu \varepsilon_{r r}, \\
\sigma_{\theta \theta}=\sigma_{\phi \phi}=\lambda\left(\varepsilon_{r r}+\varepsilon_{\theta \theta}+\varepsilon_{\phi \phi}\right)+2 \mu \varepsilon_{\theta \theta},
\end{gathered}
$$

where $\varepsilon_{r r}=\frac{d u}{d r}$ and $\varepsilon_{\theta \theta}=\varepsilon_{\phi \phi}=\frac{u}{r}$ under spherical symmetry; $u(r)$ being the radial displacement.

The potential energy of the hollow sphere, assuming a surface energy $\gamma$ at the inner surface and a pressure $P$ on the outer surface, is

$$
\begin{aligned}
W(u)= & \int_{a}^{b} 2 \pi\left(\sigma_{r r} \varepsilon_{r r}+2 \sigma_{\theta \theta} \varepsilon_{\theta \theta}\right) r^{2} d r \\
& -4 \pi b^{2} P u(b)+4 \pi \gamma[a+u(a)]^{2} .
\end{aligned}
$$

By the principle of minimum potential energy, the solution needs to satisfy $\left.\frac{d W(u+\epsilon \eta)}{d \epsilon}\right|_{\epsilon=0}$ for every admissible variation $\eta(r)$.

$$
\begin{gathered}
0=\left.\frac{\partial W}{\partial \epsilon}\right|_{\epsilon=0} \\
=\int_{a}^{b} 2 \pi r^{2}\left[\sigma_{r r}(u) \varepsilon_{r r}(\eta)+2 \sigma_{\theta \theta}(u) \varepsilon_{\theta \theta}(\eta)\right] d r \\
+\int_{a}^{b} 2 \pi r^{2}\left[\sigma_{r r}(\eta) \varepsilon_{r r}(u)+2 \sigma_{\theta \theta}(\eta) \varepsilon_{\theta \theta}(u)\right] d r
\end{gathered}
$$

*ortiz@aero.caltech.edu

${ }^{1}$ A. L. Gurson, J. Eng. Mater. Technol. 99, 2 (1977).

${ }^{2}$ A. L. Gurson, Proc. Int. Conf. Fracture A 2, 357 (1977).

${ }^{3}$ V. Tvergaard and A. Needleman, Acta Metall. 32, 157 (1984).

${ }^{4}$ J. M. Duva and J. W. Hutchinson, Mech. Mater. 3, 41 (1984).

${ }^{5}$ M. E. Mear and J. W. Hutchinson, Mech. Mater. 4, 395 (1985).

${ }^{6}$ V. Tvergaard, Adv. Appl. Mech. 27, 83 (1990).

${ }^{7}$ M. Gologanu, J. B. Leblond, and J. Devaux, J. Eng. Mater. Technol. 116, 290 (1994).

${ }^{8}$ J. B. Leblond, G. Perrin, and P. Suquet, Int. J. Plast. 10, 213 (1994).

${ }^{9}$ A. Molinari and S. Mercier, J. Mech. Phys. Solids 49, 1497 (2001).

${ }^{10}$ A. A. Benzerga and J. Besson, Eur. J. Mech. A/Solids 20, 397 (2001).

${ }^{11}$ K. Weinberg, A. Mota, and M. Ortiz, Comput. Mech. 37, 142 (2006).

${ }^{12}$ L. Xue, Int. J. Solids Struct. 44, 5163 (2007).

${ }^{13}$ K. Nahshon and J. W. Hutchinson, Eur. J. Mech. A/Solids 27, 1 (2008).

${ }^{14}$ A. Needleman and J. R. Rice, Mechanics of Sheet Metal Forming, (Plenum, New York, 1978), p. 237.

${ }^{15}$ V. Tvergaard, Int. J. Solids Structures 18, 659 (1982).

${ }^{16} \mathrm{~V}$. Tvergaard and A. Needleman, in Mechanical Behavior of Materials $I V$, edited by Janne Carlsson and N. G. Ohlson, (Pergamon, New York, 1983), p. 51.

${ }^{17}$ V. Tvergaard, J. Mech. Phys. Solids 35, 43 (1987).

${ }^{18}$ J. Pan, M. Saje, and A. Needleman, Int. J. Fract. 21, 261 (1983).

${ }^{19} \mathrm{~K}$. Weinberg and T. Böhme, IEEE Trans. Compon. Packag. Technol. 32, 684 (2009).

$$
\begin{aligned}
& -4 \pi b^{2} P \eta(b)+8 \pi \gamma[a+u(a)] \eta(a) \\
= & \int_{a}^{b} 2 \pi r^{2}\left[2 \sigma_{r r}(u) \frac{d \eta}{d r}+4 \sigma_{\theta \theta}(u) \frac{\eta}{r}\right] d r \\
& -4 \pi b^{2} P \eta(b)+8 \pi \gamma(a+u(a)) \eta(a) \\
= & -\int_{a}^{b} 4 \pi r^{2}\left[\frac{d \sigma_{r r}}{d r}+\frac{2}{r}\left(\sigma_{r r}-\sigma_{\theta \theta}\right)\right] \eta \\
& +4 \pi b^{2}\left[\sigma_{r r}(b)-P\right] \eta(b)-4 \pi a^{2} \sigma_{r r}(a) \eta(a) \\
& +8 \pi \gamma[a+u(a)] \eta(a) .
\end{aligned}
$$

The equilibrium equation and the boundary conditions are recovered.

$$
\begin{gathered}
\frac{d \sigma_{r r}}{d r}+\frac{2}{r}\left(\sigma_{r r}-\sigma_{\theta \theta}\right)=0, \quad a<r<b, \\
\sigma_{r r}(b)=P, \\
\sigma_{r r}(a)=2 \gamma \frac{a+u(a)}{a^{2}} .
\end{gathered}
$$

In the limit of a rigid material, the inner boundary condition can be simplified to

$$
\sigma_{r r}(a)=\frac{2 \gamma}{a},
$$

and the sought-after result is obtained. This pressure difference emanating from a curved surface characterized by a surface energy is very well known in fluids and the same relation holds for solid materials.
${ }^{20}$ C. Czarnota, N. Jaques, S. Mercier, and A. Molinari, J. Mech. Phys. Solids 56, 1624 (2008).

${ }^{21}$ J. Marian, J. Knap, and M. Ortiz, Phys. Rev. Lett. 93, 165503 (2004).

${ }^{22}$ D. C. Ahn, P. Sofronis, M. Kumar, J. Belak, and R. Minich, J. Appl. Phys. 101, 063514 (2007).

${ }^{23}$ S. Traiviratana, E. M. Bringa, D. J. Benson, and M. A. Meyers, Acta Mater. 56, 3874 (2008).

${ }^{24}$ M. A. Meyers, S. Traiviratana, V. A. Lubarda, D. J. Benson, and E. M. Bringa, JOM 61, 35 (2009).

${ }^{25}$ J. Belak, J. Comput.-Aided Mate.s Des. 5, 193 (1998).

${ }^{26}$ J. Belak, J. Comput.-Aided Mater. Des. 9, 165 (2002).

${ }^{27}$ R. E. Rudd and J. F. Belak, Comput. Mater. Sci. 24, 148 (2002).

${ }^{28}$ E. T. Seppälä, J. Belak, and R. E. Rudd, Phys. Rev. B 69, 134101 (2004).

${ }^{29}$ E. T. Seppälä, J. Belak, and R. E. Rudd, Phys. Rev. Lett. 93, 245503 (2004).

${ }^{30}$ L. P. Dávila, P. Erhart, E. M. Bringa, M. A. Meyers, V. A. Lubarda, M. S. Schneider, R. Becker, and M. Kumar, Appl. Phys. Lett. 86, 161902 (2005).

${ }^{31}$ R. Raj and M. F. Ashby, Acta Metall. 23, 653 (1975).

${ }^{32}$ A. Strachan, T. Cagin, and W. A. Goddard III, Phys. Rev. B 63 , 060103 (2001)

${ }^{33}$ V. Gavini, Phys. Rev. Lett. 101, 205503 (2008).

${ }^{34}$ A. B. Bortz, M. H. Kalos, and J. L. Lebowitz, J. Comput. Phys. 17, 10 (1975).

${ }^{35}$ V. Gavini, Proc. R. Soc. London, Ser. A 465, 3239 (2009). 
${ }^{36}$ G. Ho, M. T. Ong, K. J. Caspersen, and E. A. Carter, Phys. Chem. Chem. Phys. 9, 4951 (2007).

${ }^{37}$ T. Sinno, Z. K. Jiang, and R. A. Brown, Appl. Phys. Lett. 68, 3028 (1996).

${ }^{38}$ W. M. Young and E. W. Elcock, Proc. Phys. Soc. London 89, 735 (1966).

${ }^{39}$ L. C. A. La Magna and S. Coffa, Nucl. Instrum. Methods Phys. Res B 148, 262 (1999).

${ }^{40}$ B. P. Haley, K. M. Beardmore, and N. Gronbech-Jensen, Phys. Rev. B 74, 045217 (2006).

${ }^{41}$ C. L. Liu and J. B. Adams, Surf. Sci. 72, 73 (1992).

${ }^{42}$ B. P. Uberuaga, R. Smith, A. R. Cleave, F. Montalenti, G. Henkelman, R. W. Grimes, A. F. Voter, and K. E. Sickafus, Phys. Rev. Lett. 92, 115505 (2004).

${ }^{43}$ G. H. Vineyard, J. Phys. Chem. Solids 3, 121 (1957).

${ }^{44}$ F. Seitz, Phys. Rev. 74, 1513 (1948).

${ }^{45}$ A. M. Cuitino and M. Ortiz, Acta Mater. 44, 427 (1996).

${ }^{46}$ O. Penrose, J. Stat. Phys. 89, 305 (1997).

${ }^{47}$ K. E. Sickafus, E. A. Kotomin, and B. P. Uberuaga, Radiation Effects in Solids (Springer, New York, 2007).

${ }^{48}$ B. D. Lubachevsky, J. Comput. Phys. 75, 103 (1988).

${ }^{49}$ D. R. Jefferson, ACM Trans. Program. Lang. Syst. 7, 404 (1985).

${ }^{50}$ Y. Shima and J. G. Amar, Phys. Rev. B 71, 115436 (2005).

${ }^{51}$ E. Martinez, J. Marian, M. H. Kalos, and J. M. Perlado, J. Comput. Phys. 227, 3804 (2008).

${ }^{52}$ E. Martinez, J. M. Perlado, and J. Marian, J. Comput. Phys. 230, 1359 (2011).

${ }^{53}$ J. Keck, Discuss. Faraday Soc. 33, 173 (1962).

${ }^{54}$ J. H. Weiner, Statistical Mechanics of Elasticity (Dover, New York, 2002).

${ }^{55}$ M. A. Meyers, F. Gregori, B. K. Kad, M. S. Schneider, D. H. Kalantar, B. A. Remington, G. Ravichandran, T. Boehly, and J. S. Wark, Acta Mater. 51, 1211 (2003).

${ }^{56}$ D. A. Porter and K. E. Easterling, Phase Transformations in Metals and Alloys (Chapman and Hall, London, 1981).

${ }^{57}$ R. Phillips, Crystals, Defects and Microstructure: Modeling Across Scales (Cambridge University Press, Cambridge, England, 2001).

${ }^{58}$ J. Marian, J. Knap, and M. Ortiz, Acta Mater. 53, 2893 (2005).

${ }^{59}$ H. Gao, Y. Huang, and W. D. Nix, Naturwissenschaften 86, 507 (1999).

${ }^{60}$ L. E. Murr, Interfacial Phenomena in Metals and Alloys (AddisonWesley, Reading, MA, 1975).

${ }^{61}$ F. Cardarelli, Materials Handbook: A Concise Desktop Reference (Springer, New York, 2008).

${ }^{62}$ M. A. Meyers, Dynamic Behaviour of Materials (Wiley Interscience, New York, 1994).

${ }^{63} \mathrm{~V}$. Gavini, (private communication).

${ }^{64}$ W. F. Giauque and P. F. Meads, J. Am. Chem. Soc. 63, 1897 (1941).

${ }^{65}$ T. Antoun, L. Seaman, D. R. Curran, G. I. Kanel, S. V. Razorenov, and A. V. Utkin, Spall Fracture (Springer, New York, 2003).

${ }^{66}$ U. Kaiser, D. A. Muller, J. L. Grazul, A. Chuvilin, and M. Kawasaki, Nat. Mater. 1, 102 (2002).

${ }^{67}$ J. J. Gammage, D. S. Wilkinson, J. D. Embury, and E. Maire, Philos. Mag. 85, 3191 (2005).
${ }^{68}$ G. I. Kanel, S. V. Razorenov, K. Baumung, and J. Singer, J. Appl. Phys. 90, 136 (2001).

${ }^{69}$ E. Moshe, S. Eliezer, Z. Henis, M. Werdiger, E. Dekel, Y. Horovitz, S. Maman, I. B. Goldberg, and D. Eliezer, Appl. Phys. Lett. 76, 1555 (2000).

${ }^{70}$ M. A. Meyers and C. T. Aimone, Prog. Mater. Sci. 28, 1 (1983).

${ }^{71}$ E. Dekel, S. Eliezer, Z. Henis, E. Moshe, A. Ludmirsky, and I. B. Goldberg, J. Appl. Phys. 84, 4851 (1998).

${ }^{72}$ J. L. Brewer, D. A. Dalton, E. D. Jackson, A. C. Bernstein, W. Grigsby, E. M. Taleff, and T. Ditmire, Metall. Mater. Trans. A 38, 2666 (2007).

${ }^{73}$ X. Chen, J. R. Asay, S. K. Dwivedi, and D. P. Field, J. Appl. Phys. 99, 023528 (2006).

${ }^{74}$ D. A. Dalton, J. Brewer, A. C. Bernstein, W. Grigsby, D. Milathianaki, E. Jackson, R. Adams, P. Rambo, J. Schwarz, A. Edens, M. Geissel, I. Smith, E. Taleff, and T. Ditmire, AIP Conference Proceedings 955, 501 (2007).

${ }^{75}$ J. P. Cuq-Lelandais, M. Boustie, L. Berthe, T. de Resseguier, P. Combis, J. P. Combis, J. P. Colombier, M. Nivard, and A. Claverie, J. Phys. D 42, 065402 (2009).

${ }^{76}$ D. E. Grady, J. Mech. Phys. Solids 36, 353 (1988).

${ }^{77}$ G. I. Kanel, S. V. Razorenov, A. Bogatch, A. V. Utkin, and V. E. F. and D. E. Grady, J. Appl. Phys. 79, 8310 (1996).

${ }^{78}$ G. I. Kanel, S. V. Razorenov, A. V. Utkin, K. Bauming, H. U. Karow, and V. Licht, AIP Conf. Proc. 309, 1043 (1994).

${ }^{79}$ E. Moshe, S. Eliezer, E. Dekel, A. Ludmirsky, Z. Henis, M. Werdiger, I. B. Goldberg, N. Eliaz, and D. Eliezer, J. Appl. Phys. 83, 4004 (1998).

${ }^{80}$ V. E. Fortov, V. V. Kostin, and S. Eliezer, J. Appl. Phys. 70, 4524 (1991).

${ }^{81}$ S. Hanim and J. R. Klepaczko, Int. J. Impact Eng. 22, 649 (1999).

${ }^{82}$ N. W. Ashcroft and N. D. Mermin, Solid State Physics (Saunders, Philadelphia, 1976).

${ }^{83}$ This is indeed the case at zero pressure (Ref. 93).

${ }^{84}$ E. M. Bringa, K. Rosolankova, R. E. Rudd, B. A. Remington, J. S. Wark, M. Duchaineau, D. H. Kalantar, J. Hawreliak, and J. Belak, Nat. Mater. 5, 805 (2006).

${ }^{85}$ M. A. Meyers, O. Vohringer, and V. A. Lubarda, Acta Mater. 49, 4025 (2001).

${ }^{86}$ J. W. Christian and S. Mahajan, Prog. Mater. Sci. 39, 1 (1995).

${ }^{87}$ S. G. Song and G. T. G. III, Metall. Mater. Trans. A 26, 2665 (1995).

${ }^{88}$ M. Chen, E. Ma, K. J. Hemker, H. Sheng, Y. Wang, and X. Cheng, Science 300, 1275 (2003).

${ }^{89}$ Q. F. Guan, Q. Y. Zhang, C. Dong, and G. T. Zou, J. Mater. Sci. 40, 5049 (2005).

${ }^{90}$ Y. T. Zhu, Nanostructured Materials by High-Pressure Severe Plastic Deformation (Springer, Netherland, 2006), Vol. 212, p. 3.

${ }^{91}$ A. Lo and R. T. Skodje, J. Chem. Phys. 112, 1966 (2000).

${ }^{92}$ J. Dai, W. D. Seider, and T. Sinno, Mol. Simul. 32, 305 (2006).

${ }^{93}$ P. J. H. Denteneer and J. M. Soler, J. Phys.: Condens. Matter 3, 8777 (1991).

${ }^{94}$ G. Ho, M. T. Ong, K. J. Caspersen, and E. A. Carter, Phys. Chem. Chem. Phys. 9, 4951 (2007). 